\title{
COMMUNICATION
}

\section{Intoxication au paracétamol et douleurs dentaires: revue de la littérature et recommandations de prise en charge.}

\section{Lafont $\mathrm{J}^{1}$, Lan $\mathrm{R}^{1,2}$, Roche-Poggi $\mathrm{P}^{1}$, Campana F3, Catherine $\mathrm{JH}^{1,2}$}

1. Service d'Odontologie, Département de Chirurgie Orale - Timone, AP-HM - Marseille

2. UMR 7268 ADES, Aix-Marseille/EFS/CNRS - Faculté de médecine secteur Nord - AMU, AP-HM - Marseille

3. Centre Massilien de la Face - Marseille

\section{Introduction}

Les douleurs liées aux affections ou aux soins dentaires sont très courantes dans la population générale aussi bien adulte que pédiatrique et peuvent être particulièrement invalidantes. Le paracétamol est l'antalgique le plus couramment utilisé pour les traiter, sur prescription médicale comme en automédication. En France, les intoxications médicamenteuses constituent un problème de santé publique. Le paracétamol est responsable de 2 à $7 \%$ des intoxications médicamenteuses. Il est la deuxième molécule la plus retrouvée dans les intoxications médicamenteuses après les benzodiazépines.

\section{Matériel et méthodes}

Une revue de la littérature a été réalisée en consultant les bases de données Pubmed et Web of science de 1986 à 2016 avec les mots clés « overdose AND paracetamol AND dental pain ».

\section{Résultats}

Peu de publications sur le sujet sont retrouvées, moins d'une dizaine ont parues pertinentes. Parmi elles une étude cas-témoins, une étude rétrospective et cinq case-reports. Au Royaume-Uni les douleurs dentaires sont la première cause d'intoxication aiguë involontaire au paracétamol. Les patients concernés n'avaient pour la plus part pas vu de praticien ou n'avaient pas eu de rendez-vous assez vite, et avaient donc pris le paracétamol en automédication. Tous les patients ont survécu à l'intoxication. Les présentations cliniques étaient des douleurs abdominales, vomissements et acouphènes. II pouvait ne pas y avoir de symptômes du tout dans les vingtquatre premières heures. Les stigmates biologiques étaient ceux d'une insuffisance hépatique aiguë pouvant aller jusqu'à la nécrose hépatique. Le seuil critique au-delà duquel apparaissent les signes cliniques ou biologiques était de 10 à $15 \mathrm{~g}$ de paracétamol par $24 \mathrm{~h}$ soit une posologie de $150 \mathrm{à} 250 \mathrm{mg} / \mathrm{kg}$ environ. Si ce seuil est dépassé, le traitement par antidote du paracétamol (N-Acétyl-Cystéine ou NAC) doit être initié le plus rapidement possible, et si l'on intervient moins d'une heure après l'ingestion il pourra être associé à un lavage gastrique. La surveillance est clinique mais aussi biologique comprenant le bilan hépatique et la paracétamolémie.

\section{Conclusion}

Les douleurs dentaires sont la première cause d'intoxication aiguë involontaire au paracétamol. Dans la majorité des cas elles surviennent en contexte d'automédication, chez des patients qui n'ont pas pu ou n'ont pas cherché à voir de praticien. L'existence de médicaments combinant le paracétamol à d'autres molécules antalgiques est un facteur de risque important de surdosage, car les patients associent souvent plusieurs médicaments contenant du paracétamol sans le savoir. Ces études montrent l'importance pour les praticiens de bien connaître les médicaments que prennent couramment leurs patients. Elles montrent aussi l'importance cruciale de l'interrogatoire dans l'évaluation du risque de surdosage en paracétamol, ainsi que la nécessité d'accès facile à des soins dentaires rapides pour les patients.

jacinthelafont@gmail.com

\section{Références}

Nayyer, N. V., J. Byers, and C. Marney. "Identifying Adults at Risk of Paracetamol Toxicity in the Acute Dental Setting: Development of a Clinical Algorithm." British Dental Journal 216, no. 5 (March 7, 2014): 229-35. doi:10.1038/sj.bdj.2014.146. Siddique, I., H. Mahmood, and R. Mohammed-Ali. "Paracetamol Overdose Secondary to Dental Pain: A Case Series." British Dental Journal 219, no. 6 (September 25, 2015): E6-E6. doi:10.1038/ sj.bdj.2015.706.

(C) The authors, published by EDP Sciences. This is an Open Access article distributed under the terms of the Creative Commons Attribution License 4.0 (http://creativecommons.org/licenses/by/4.0/). 